\title{
ARCHIMEDEAN UNITAL GROUPS WITH FINITE UNIT INTERVALS
}

\author{
DAVID J. FOULIS
}

\author{
Received 23 October 2002
}

\begin{abstract}
Let $G$ be a unital group with a finite unit interval $E$, let $n$ be the number of atoms in $E$, and let $\kappa$ be the number of extreme points of the state space $\Omega(G)$. We introduce canonical order-preserving group homomorphisms $\xi: \mathbb{Z}^{n} \rightarrow G$ and $\rho: G \rightarrow \mathbb{Z}^{\kappa}$ linking $G$ with the simplicial groups $\mathbb{Z}^{n}$ and $\mathbb{Z}^{\kappa}$. We show that $\xi$ is a surjection and $\rho$ is an injection if and only if $G$ is torsion-free. We give an explicit construction of the universal group (unigroup) for $E$ using the canonical surjection $\xi$. If $G$ is torsion-free, then the canonical injection $\rho$ is used to show that $G$ is Archimedean if and only if its positive cone is determined by a finite number of homogeneous linear inequalities with integer coefficients.
\end{abstract}

2000 Mathematics Subject Classification: 06F20.

1. Introduction and basic definitions. In this paper, we continue the study of unital groups with finite unit intervals begun in [2, 3]. Motivation for this study can be found in [2]. Although we will attempt to keep this paper somewhat self-contained, we make free use of the notation, nomenclature, and results of $[2,3]$.

We begin by setting forth notation and recalling some basic definitions. We write a partially ordered abelian group $G$ additively, and denote the positive cone in $G$ by $G^{+}:=\{g \in G \mid 0 \leq g\}$ [7]. If $G^{+}$generates $G$ as an abelian group, that is, if $G=G^{+}-G^{+}$, then $G$ is said to be directed. A subset $F$ of $G^{+}$is cone generating if and only if every element of $G^{+}$is a sum of a finite sequence of (not necessarily distinct) elements of $F$. Various definitions of "Archimedean" can be found in the literature. We use the following [7, page 20]: $G$ is Archimedean if and only if, for $a, b \in G$, the condition $n a \leq b$ for every positive integer $n$ implies that $a \leq 0$.

If $G$ is a partially ordered abelian group and $u \in G^{+}$, we define the interval $E:=G^{+}[0, u]:=\{g \in G \mid 0 \leq g \leq u\}$. Thus $E$ forms a bounded partially ordered set under the restriction of the partial order $\leq$ on $G$ to $E$. The interval $E$ can be organized into an effect algebra under the partial binary operation $\oplus$ obtained by restriction of + to $E$. For the details see $[1,5]$.

An element $u \in G^{+}$is called an order unit if and only if each element of $G$ is dominated by a positive integer multiple of $u$ [7, page 4]. A unital group [2] is a partially ordered abelian group $G$ with a specified order unit $u$, called the unit, such that the interval $E:=G^{+}[0, u]$, called the unit interval, is cone generating. 
If $G$ is a unital group with unit $u$, then $G$ is directed, and $G \neq\{0\} \Leftrightarrow u \neq 0$. If $G$ is directed, $u \in G^{+}$, and $G^{+}[0, u]$ is cone generating, then $G$ is a unital group with unit $u$.

If $G$ is a unital group with unit interval $E$, and if $K$ is an abelian group, then a mapping $\phi: E \rightarrow K$ is called a $K$-valued measure on $E$ if and only if $p, q, p+q \in E \Rightarrow \phi(p+q)=\phi(p)+\phi(q)$. For instance, if $\Phi: G \rightarrow K$ is a group homomorphism, then the restriction $\phi:=\left.\Phi\right|_{E}$ of $\Phi$ to $E$ is a $K$-valued measure on $E$. If every $K$-valued measure on $E$ is the restriction to $E$ of a group homomorphism from $G$ to $K$, then $G$ is called a $K$-unital group. A unigroup is a unital group that is $K$-unital for every abelian group $K[5,6]$.

Let $G$ and $H$ be unital groups with unit intervals $E$ and $L$, respectively, and units $u$ and $v$, respectively. A mapping $\phi: E \rightarrow L$ is an effect-algebra morphism [5, Definition 6.1] if and only if $\phi(u)=v$ and, regarded as a mapping $\phi: E \rightarrow$ $H$, it is an $H$-valued measure. Let $\phi: E \rightarrow L$ be an effect-algebra morphism. If $p_{i} \in E, k_{i}$ are nonnegative integers for $i=1,2, \ldots, n$, and $\sum_{i=1}^{n} k_{i} p_{i} \in E$, then $\phi\left(\sum_{i=1}^{n} k_{i} p_{i}\right)=\sum_{i=1}^{n} k_{i} \phi\left(p_{i}\right) \in L$. If $\phi: E \rightarrow L$ is a bijective effect-algebra morphism and $\phi^{-1}: L \rightarrow E$ is also an effect-algebra morphism, then $\phi: E \rightarrow L$ is an effect-algebra isomorphism.

We use the usual notation $\mathbb{R}, \mathbb{Q}$, and $\mathbb{Z}$ for the ordered field of real numbers, the ordered field of rational numbers, and the ordered ring of integers, respectively. Thus, the standard positive cone in $\mathbb{R}$ is $\mathbb{R}^{+}:=\left\{x^{2} \mid x \in \mathbb{R}\right\}$ and the standard positive cones in $\mathbb{Q}$ and $\mathbb{Z}$ are $\mathbb{Q}^{+}:=\mathbb{Q} \cap \mathbb{R}^{+}$and $\mathbb{Z}^{+}:=\mathbb{Z} \cap \mathbb{Q}^{+}$. We often disregard the multiplicative structures of $\mathbb{R}, \mathbb{Q}$, and $\mathbb{Z}$ and regard them as partially ordered additive abelian groups. As such, and with 1 as the unit, $\mathbb{R}, \mathbb{Q}$, and $\mathbb{Z}$ provide examples of unigroups.

Let $G \neq\{0\}$ be a unital group with unit $u$ and unit interval $E$. Then a state for $G$ is a group homomorphism $\omega: G \rightarrow \mathbb{R}$ such that $\omega\left(G^{+}\right) \subseteq \mathbb{R}^{+}$and $\omega(u)=1$. The set of all states for $G$, called the state space of $G$, is denoted by $\Omega(G)$. By [7, Corollary 4.4, Proposition 6.2], $\Omega(G)$ is a nonempty compact convex subset of the locally convex Hausdorff linear topological space $\mathbb{R}^{G}$ with the topology of pointwise convergence. If $\omega \in \Omega(G)$ and $\omega(G) \subseteq \mathbb{Q}$, then $\omega$ is called a $\mathbb{Q}$-valued state. Evidently, $\omega \in \Omega(G)$ is $\mathbb{Q}$-valued if and only if $\omega(E) \subseteq \mathbb{Q}^{+}$. A probability measure on $E$ is an $\mathbb{R}$-valued measure $\pi$ on $E$ such that $\pi(E) \subseteq[0,1] \subseteq \mathbb{R}$ and $\pi(u)=1$. The restriction to $E$ of a state $\omega \in \Omega(G)$ is a probability measure on $E$. If $G$ is $\mathbb{R}$-unital, then each probability measure $\pi$ on $E$ is the restriction to $E$ of a uniquely determined state $\omega \in \Omega(G)$.

Let $\Delta \subseteq \Omega(G)$. Then $\Delta$ is said to be strictly positive if and only if, for each $0 \neq p \in G^{+}$, there exists $\omega \in \Delta$ with $0<\omega(p)$. If $\omega \in \Omega(G)$, and $\{\omega\}$ is strictly positive, we say that $\omega$ is a strictly positive state. If $G^{+}=\{p \in G \mid$ $0 \leq \omega(p)$ for all $\omega \in \Delta\}$, then $\Delta$ is said to be cone determining. By [7, Theorem 4.14], $G$ is Archimedean if and only if $\Omega(G)$ is cone determining. By definition, $\Delta$ is separating if and only if, for all $g \neq 0 \in G$, there exists $\omega \in \Delta$ with $\omega(g) \neq 0$. 
If $r$ is a positive integer, we understand that $\mathbb{R}^{r}, \mathbb{Q}^{r}$, and $\mathbb{Z}^{r}$ are organized into additive abelian groups with coordinatewise operations. The standard partial order for each of these groups is the coordinatewise partial order determined by the standard total orders on $\mathbb{R}, \mathbb{Q}$, and $\mathbb{Z}$, and the corresponding standard positive cones are $\left(\mathbb{R}^{+}\right)^{r},\left(\mathbb{Q}^{+}\right)^{r}$, and $\left(\mathbb{Z}^{+}\right)^{r}$.

With the standard partial order, $\mathbb{Z}^{r}$ forms the so-called simplicial group [7, page 47]. As a simplicial group, $\mathbb{Z}^{r}$ is an Archimedean lattice-ordered group with the smallest order unit, namely $(1,1, \ldots, 1)$. An element $\mathbf{v} \in\left(\mathbb{Z}^{+}\right)^{r}$ is an order unit if and only if all of its coordinates are strictly positive. If $\mathbf{v}$ is an order unit in the simplicial group $\mathbb{Z}^{r}$, then the unit interval $\left(\mathbb{Z}^{+}\right)^{r}[\mathbf{0}, \mathbf{v}]$ forms a finite MV-algebra [4]. Conversely, every finite MV-algebra has this form. If $\mathbf{v}=(1,1, \ldots, 1)$, then $\left(\mathbb{Z}^{+}\right)^{r}[\mathbf{0}, \mathbf{v}]$ is isomorphic to the finite Boolean algebra $2^{r}$.

This paper is focused on the study of a unital group $G$ with a finite unit interval $E$ and on the question of just when $G$ is Archimedean (i.e., carries a cone-determining set of states). If $G$ carries a cone-determining set of states, then it is clear that $G$ is torsion-free, that is, 0 is the only element of finite order in $G$, so in Sections 4 and 5 we will be paying special attention to the torsion-free case. If $G \neq\{0\}$ is a unital group with a finite unit interval $E$, then there are atoms (minimal nonzero elements) in $E$, and every nonzero element in $E$ dominates at least one atom. Thus, we will be working with the following data.

1.1. Standing assumptions and notation. For the remainder of this paper, $G$ is a unital group with unit $u \neq 0$ and with a finite unit interval $E=G^{+}[0, u]$. The distinct atoms in $E$ are denoted by $a_{1}, a_{2}, \ldots, a_{n}$.

By [2, Lemma 5.1], the finite set $\left\{a_{1}, a_{2}, \ldots, a_{n}\right\} \subseteq E \subseteq G^{+}$is cone generating, and since $G$ is directed, $\left\{a_{1}, a_{2}, \ldots, a_{n}\right\}$ is a finite set of generators for the abelian group $G$. By the fundamental theorem for finitely generated abelian groups, the torsion subgroup $G_{\tau}$ of $G$ is finite and $G$ is a direct sum of $G_{T}$ and a torsion-free subgroup $H \subseteq G$ of finite rank $r>0$. If $\eta: G \rightarrow H$ is the natural projection homomorphism with $\operatorname{ker}(\eta)=G_{\tau}$, then by [2, Theorem 4.1] $H$ can be organized into a unital group with unit $\eta(u)$, with positive cone $H^{+}=\eta\left(G^{+}\right)$, and with a finite unit interval $L$. Moreover, there is an affine isomorphism $\omega \mapsto$ $\widetilde{\omega}$ from $\Omega(G)$ onto $\Omega(H)$ such that $\omega=\widetilde{\omega} \circ \eta$ for all $\omega \in \Omega(G)$. As $H$ is a torsion-free group of finite rank $r$, there is a group isomorphism $\phi: H \rightarrow \mathbb{Z}^{r}$ and, by [3, Lemma 3.2], $\phi$ can be chosen in such a way that $\phi\left(H^{+}\right) \subseteq\left(\mathbb{Z}^{+}\right)^{r}$. By [2, Lemma 3.5], the set of $\mathbb{Q}$-valued states on $H$ is separating.

2. The canonical surjection $\xi$. In this section, we introduce a surjective order-preserving group homomorphism $\xi$ from the simplicial group $\mathbb{Z}^{n}$ onto the unital group $G$. Recall that if $Z$ is a free abelian group, $B \subset Z$ is a free basis for $Z, K$ is an abelian group, and $f: B \rightarrow K$ is a function, then there is a unique group homomorphism $\phi: Z \rightarrow K$ that agrees with $f$ on $B$. 
Definition 2.1. Let $\mathbf{d}_{1}=(1,0, \ldots, 0), \mathbf{d}_{2}=(0,1, \ldots, 0), \ldots, \mathbf{d}_{n}=(0,0, \ldots, 1)$ be the standard free basis for the additive abelian group $\mathbb{Z}^{n}$. Define the canonical surjection $\xi: \mathbb{Z}^{n} \rightarrow G$ to be the uniquely determined group homomorphism $\xi: \mathbb{Z}^{n} \rightarrow G$ such that $\xi\left(\mathbf{d}_{i}\right)=a_{i}$ for $i=1,2, \ldots, n$.

LEMMA 2.2. (i) If $\mathbf{x}=\left(x_{1}, x_{2}, \ldots, x_{n}\right) \in \mathbb{Z}^{n}$, then $\xi(\mathbf{x})=\sum_{i=1}^{n} x_{i} a_{i}$.

(ii) The mapping $\xi: \mathbb{Z}^{n} \rightarrow G$ satisfies $G^{+}=\xi\left(\left(\mathbb{Z}^{+}\right)^{n}\right)$.

(iii) The mapping $\xi: \mathbb{Z}^{n} \rightarrow G$ satisfies $G=\xi\left(\mathbb{Z}^{n}\right)$.

(iv) The mapping $\xi: \mathbb{Z}^{n} \rightarrow G$ is a surjective order-preserving group homomorphism and its kernel satisfies the condition $\operatorname{ker}(\xi) \cap\left(\mathbb{Z}^{+}\right)^{n}=\{\mathbf{0}\}$.

Proof. Part (i) follows immediately from Definition 2.1. Part (ii) follows from (i) and the fact that $\left\{a_{1}, a_{2}, \ldots, a_{n}\right\}$ is a cone-generating set. Part (iii) follows from (ii) and the fact that $G$ is directed. To prove (iv), we begin by observing that the group homomorphism $\xi: \mathbb{Z}^{n} \rightarrow G$ is order preserving by (ii) and surjective by (iii). Suppose $\mathbf{x}=\left(x_{1}, x_{2}, \ldots, x_{n}\right) \in \operatorname{ker}(\xi) \cap\left(\mathbb{Z}^{+}\right)^{n}$. Then $\sum_{i=1}^{n} x_{i} a_{i}=0$ and $0 \leq x_{i}$ for $i=1,2, \ldots, n$. Since $x_{i} a_{i} \in G^{+}$for $i=1,2, \ldots, n$, it follows that $x_{i} a_{i}=0$ for $i=1,2, \ldots, n$. Therefore, since $0 \neq a_{i} \in G^{+}$, we have $x_{i}=0$ for $i=1,2, \ldots, n$.

DEFINITION 2.3. Define $T:=\xi^{-1}(u) \cap\left(\mathbb{Z}^{+}\right)^{n}$. Vectors $\mathbf{t} \in T$ are called multiplicity vectors for $G$ (cf. [2, Definition 5.2]). Define $\mathbb{D}$ to be the subgroup of $\mathbb{Z}^{n}$ generated by the set of all differences $\mathbf{t}-\mathbf{s}$ for $\mathbf{t}, \mathbf{s} \in T$, let $G^{*}$ be the quotient group $\mathbb{Z}^{n} / \mathbb{D}$, and let $\xi^{*}: \mathbb{Z}^{n} \rightarrow G^{*}$ be the natural surjective group homomorphism with $\operatorname{ker}\left(\xi^{*}\right)=\mathbb{D}$.

LEMMA 2.4. (i) If $\mathbf{s}, \mathbf{t} \in T$, then $\mathbf{s} \leq \mathbf{t} \Rightarrow \mathbf{s}=\mathbf{t}$.

(ii) The set $T$ is finite and nonempty.

(iii) If $\mathbf{x} \in\left(\mathbb{Z}^{+}\right)^{n}$, then $\xi(\mathbf{x}) \in E \Leftrightarrow \exists \mathbf{t} \in T, \mathbf{x} \leq \mathbf{t}$.

Proof. (i) If $\mathbf{s}, \mathbf{t} \in T$ and $\mathbf{s} \leq \mathbf{t}$, then $\mathbf{t}-\mathbf{s} \in \operatorname{ker}(\xi) \cap\left(\mathbb{Z}^{+}\right)^{n}$, so $\mathbf{s}=\mathbf{t}$ by Lemma 2.2(iv).

(ii) By (i), $T$ forms an antichain in the positive cone $\left(\mathbb{Z}^{+}\right)^{n}$ of the simplicial group $\mathbb{Z}^{n}$, hence $T$ is a finite set [8].

(iii) Let $\mathbf{x} \in\left(\mathbb{Z}^{+}\right)^{n}$. If $\mathbf{t} \in T$ and $\mathbf{x} \leq \mathbf{t}$, then $0 \leq \xi(\mathbf{x}) \leq \xi(\mathbf{t})=u$, so $\xi(\mathbf{x}) \in E$. Conversely, suppose $\xi(\mathbf{x}) \in E$. Then $u-\xi(\mathbf{x}) \in G^{+}$, so there exists $\mathbf{y} \in\left(\mathbb{Z}^{+}\right)^{n}$ with $\xi(\mathbf{y})=u-\xi(\mathbf{x})$. Therefore, $\mathbf{x}+\mathbf{y} \in\left(\mathbb{Z}^{+}\right)^{n}$ with $\xi(\mathbf{x}+\mathbf{y})=u$, and it follows that $\mathbf{t}:=\mathbf{x}+\mathbf{y} \in T$ with $\mathbf{x} \leq \mathbf{t}$.

DEFINITION 2.5. Let $T=\left\{\mathbf{t}_{1}, \mathbf{t}_{2}, \ldots, \mathbf{t}_{m}\right\}$. Then the $m \times n$ matrix $\left[t_{i j}\right]$ with $\mathbf{t}_{1}, \mathbf{t}_{2}, \ldots, \mathbf{t}_{m}$ as its successive row vectors is called the multiplicity matrix for $G$. The $m \times(n+1)$ matrix $M$ obtained by appending a final column to the matrix $\left[t_{i j}\right]$ consisting entirely of -1 's is called the relation matrix for $G$ (cf. [2, Definition 5.3]).

The relation matrix $M$ encodes $m$ fundamental relations $\sum_{j=1}^{n} t_{i j} a_{j}-u=0$ for $i=1,2, \ldots, m$ satisfied by the generators $a_{j}$ for $G$ and the unit $u$ (see [2, Theorem 5.4]). 
THEOREM 2.6. (i) $\operatorname{rank}(T)=\operatorname{rank}(M)$.

(ii) $\operatorname{rank}(G)+\operatorname{rank}(T) \leq n+1$.

(iii) If $G$ is torsion-free, then $G$ is $\mathbb{R}$-unital if and only if $\operatorname{rank}(G)+\operatorname{rank}(T)=$ $n+1$.

Proof. We will prove that the last column of $M$ is a rational linear combination of its first $n$ columns, from which (i) follows. There is a $\mathbb{Q}$-valued state $\omega \in \Omega(G)$. Let $q_{j}:=-\omega\left(a_{j}\right)$ for $j=1,2, \ldots, n$. As $\sum_{i=1}^{n} t_{i j} a_{j}=u$ for $i=1,2, \ldots, m$, we have $\sum_{i=1}^{n} t_{i j} q_{j}=-\omega(u)=-1$ for $i=1,2, \ldots, m$. Parts (ii) and (iii) now follow from [2, Theorem 5.6].

THEOREM 2.7. The abelian group $G^{*}$ can be organized into a unigroup with unit $u^{*}:=\xi^{*}(\mathbf{t})$, independent of the choice of $\mathbf{t} \in T$, and with positive cone $\left(G^{*}\right)^{+}:=\xi^{*}\left(\left(\mathbb{Z}^{+}\right)^{n}\right)$. Then there is an effect-algebra isomorphism $p \mapsto p^{*}$ from $E$ onto the unit interval $E^{*}:=\left(G^{*}\right)^{+}\left[0, u^{*}\right]$, and $\xi^{*}$ is the canonical surjection for $G^{*}$. Moreover, there is a surjective order-preserving group homomorphism $\beta: G^{*} \rightarrow G$ such that $\beta\left(\left(G^{*}\right)^{+}\right)=G^{+}, \beta\left(p^{*}\right)=p$ for all $p \in E$, and $\xi=\beta \circ \xi^{*}$.

Proof. Evidently, $\mathbb{D} \subseteq \operatorname{ker}(\xi)$, whence $\mathbb{D} \cap\left(\mathbb{Z}^{+}\right)^{n}=\{\mathbf{0}\}$ by Lemma 2.2(iv), so $G^{*}$ can be organized into a partially ordered abelian group with positive cone $\left(G^{*}\right)^{+}:=\xi^{*}\left(\left(\mathbb{Z}^{+}\right)^{n}\right)$. Since the simplicial group $\mathbb{Z}^{n}$ is directed and $\xi^{*}: \mathbb{Z}^{n} \rightarrow G^{*}$ is a surjective order-preserving group homomorphism, it follows that $G^{*}$ is directed. Clearly, $u^{*}:=\xi^{*}(\mathbf{t})$ is independent of the choice of $\mathbf{t} \in T$, whence $u^{*} \in\left(G^{*}\right)^{+}$.

We claim that if $\mathbf{x} \in\left(\mathbb{Z}^{+}\right)^{n}$, then

$$
\xi(\mathbf{x}) \in E \Longleftrightarrow \xi^{*}(\mathbf{x}) \in E^{*} .
$$

To prove (2.1), suppose $\mathbf{x} \in\left(\mathbb{Z}^{+}\right)^{n}$. If $\xi(\mathbf{x}) \in E$, then by Lemma 2.4(iii) there exists $\mathbf{t} \in T$ with $\mathbf{x} \leq \mathbf{t}$, whence $0 \leq \xi^{*}(\mathbf{x}) \leq \xi^{*}(\mathbf{t})=u^{*}$, so $\xi^{*}(\mathbf{x}) \in\left(G^{*}\right)^{+}\left[0, u^{*}\right]=$ $E^{*}$. Conversely, if $\xi^{*}(\mathbf{x}) \in E^{*}$, there exists $\mathbf{y} \in\left(\mathbb{Z}^{+}\right)^{n}$ such that $u^{*}-\xi^{*}(\mathbf{x})=$ $\xi^{*}(\mathbf{y})$, whence $\xi^{*}(\mathbf{x}+\mathbf{y})=\xi^{*}(\mathbf{t})$ for any choice of $\mathbf{t} \in T, \mathbf{x}+\mathbf{y}-\mathbf{t} \in \mathbb{D} \subseteq \operatorname{ker}(\xi)$, and $\xi(\mathbf{x})+\xi(\mathbf{y})=\xi(\mathbf{t})=u$. Therefore, $\xi(\mathbf{x}) \in E$, and (2.1) follows.

For each $i=1,2, \ldots, n$, we have $\mathbf{d}_{i} \in\left(\mathbb{Z}^{+}\right)^{n}$ with $\xi\left(\mathbf{d}_{i}\right)=a_{i} \in E$, whence by (2.1), $\xi^{*}\left(\mathbf{d}_{i}\right) \in E^{*}$. Since $\left(\mathbb{Z}^{+}\right)^{n}$ is the set of all linear combinations of $\mathbf{d}_{1}, \mathbf{d}_{2}, \ldots$, $\mathbf{d}_{n}$ with nonnegative integer coefficients, it follows that $\left\{\xi^{*}\left(\mathbf{d}_{i}\right) \mid i=1,2, \ldots, n\right\}$ is a finite cone-generating subset of $E^{*}$ in $G^{*}$. Therefore, $u^{*}$ is an order unit in $\left(G^{*}\right)^{+}$and $G^{*}$ is a unital group with unit $u^{*}$ and unit interval $E^{*}$.

We claim that if $\mathbf{x}, \mathbf{y} \in\left(\mathbb{Z}^{+}\right)^{n}$, then

$$
\xi(\mathbf{x})=\xi(\mathbf{y}) \in E \Longleftrightarrow \xi^{*}(\mathbf{x})=\xi^{*}(\mathbf{y}) \in E^{*} .
$$

To prove (2.2), suppose $\mathbf{x}, \mathbf{y} \in\left(\mathbb{Z}^{+}\right)^{n}$. If $\xi(\mathbf{x})=\xi(\mathbf{y}) \in E$, then there exists $\mathbf{z} \in$ $\left(\mathbb{Z}^{+}\right)^{n}$ such that $u-\xi(\mathbf{x})=\xi(\mathbf{z})$, whence $\mathbf{x}+\mathbf{z} \in T$ and likewise $\mathbf{y}+\mathbf{z} \in T$, so $\mathbf{x}-\mathbf{y}=(\mathbf{x}+\mathbf{z})-(\mathbf{y}+\mathbf{z}) \in \mathbb{D}=\operatorname{ker}\left(\xi^{*}\right)$, and $\xi^{*}(\mathbf{x})=\xi^{*}(\mathbf{y}) \in E^{*}$ by (2.1). 
Conversely, if $\xi^{*}(\mathbf{x})=\xi^{*}(\mathbf{y}) \in E^{*}$, then $\mathbf{x}-\mathbf{y} \in \operatorname{ker}\left(\xi^{*}\right)=\mathbb{D} \subseteq \operatorname{ker} \xi$, so $\xi(\mathbf{x})=$ $\xi(\mathbf{y}) \in E$ by (2.1), proving (2.2).

If $p \in E$, we define $p^{*} \in E^{*}$ as follows: choose $\mathbf{x} \in\left(\mathbb{Z}^{+}\right)^{n}$ with $p=\xi(\mathbf{x})$ and let $p^{*}:=\xi^{*}(\mathbf{x})$. By (2.2), $p^{*}$ is well defined and the mapping $*: E \rightarrow E^{*}$ given by $p \mapsto p^{*}$ is a bijection. Evidently, this notation is consistent with $u^{*}=\xi^{*}(\mathbf{t})$, $\mathbf{t} \in T$, as defined previously. Because $\operatorname{ker}\left(\xi^{*}\right)=\mathbb{D} \subseteq \operatorname{ker}(\xi)$, there is a uniquely determined surjective group homomorphism $\beta: G^{*} \rightarrow G$ such that $\beta \circ \xi^{*}=\xi$. Clearly, $\beta\left(\left(G^{*}\right)^{+}\right)=\xi\left(\left(\mathbb{Z}^{+}\right)^{n}\right)=G^{+}$, so $\beta$ is order preserving. If $h \in E^{*}$, there exists $\mathbf{x} \in\left(\mathbb{Z}^{+}\right)^{n}$ with $h=\xi^{*}(\mathbf{x})$, whence $\beta(h)=\beta\left(\xi^{*}(\mathbf{x})\right)=\xi(\mathbf{x}) \in E$ by (2.1) and $(\beta(h))^{*}=\xi^{*}(\mathbf{x})=h$. If $p \in E$, there exists $\mathbf{x} \in\left(\mathbb{Z}^{+}\right)^{n}$ with $p=\xi(\mathbf{x}) \in E$, whence $p^{*}=\xi^{*}(\mathbf{x}) \in E^{*}$ with $\beta\left(p^{*}\right)=\beta\left(\xi^{*}(\mathbf{x})\right)=\xi(\mathbf{x})=p$. Therefore, the restriction $\left.\beta\right|_{E^{*}}$ of $\beta$ to $E^{*}$ is a bijective effect-algebra morphism of $E^{*}$ onto $E$ with $*: E \rightarrow E^{*}$ as its inverse.

Suppose $p, q, p+q \in E$ and choose $\mathbf{x}, \mathbf{y} \in\left(\mathbb{Z}^{+}\right)^{n}$ with $p=\xi(\mathbf{x})$ and $q=\xi(\mathbf{y})$. Then $\mathbf{x}+\mathbf{y} \in\left(\mathbb{Z}^{+}\right)^{n}$ with $\xi(\mathbf{x}+\mathbf{y})=p+q \in E$. Therefore, $(p+q)^{*}=\xi^{*}(\mathbf{x}+$ $\mathbf{y})=\xi^{*}(\mathbf{x})+\xi^{*}(\mathbf{y})=p^{*}+q^{*}$, so ${ }^{*}: E \rightarrow E^{*}$ is an effect-algebra morphism. Consequently, both ${ }^{*}: E \rightarrow E^{*}$ and its inverse $\left.\beta\right|_{E^{*}}: E^{*} \rightarrow E$ are effect-algebra isomorphisms.

Because $^{*}: E \rightarrow E^{*}$ is an effect-algebra isomorphism, the atoms in $E^{*}$ are $a_{i}^{*}$ for $i=1,2, \ldots, n$. Also, $\xi^{*}\left(\mathbf{d}_{i}\right)=a_{i}^{*}$ for $i=1,2, \ldots, n$, and it follows that $\xi^{*}$ is the canonical surjection for $G^{*}$.

To prove that $G^{*}$ is a unigroup, suppose that $K$ is an abelian group and $\phi$ : $E^{*} \rightarrow K$ is a $K$-valued measure. Define $\Phi: \mathbb{Z}^{n} \rightarrow K$ to be the unique group homomorphism such that $\Phi\left(\mathbf{d}_{i}\right)=\phi\left(a_{i}^{*}\right)$ for $i=1,2, \ldots, n$. Suppose $\mathbf{t}=\left(t_{1}, t_{2}, \ldots, t_{n}\right)$ $\in T$. Thus, $u=\sum_{i=1}^{n} t_{i} a_{i}$, whence $u^{*}=\sum_{i=1}^{n} t_{i} a_{i}^{*}$, and since $\phi$ is a $K$-valued measure, $\phi\left(u^{*}\right)=\sum_{i=1}^{n} t_{i} \phi\left(a_{i}^{*}\right)=\sum_{i=1}^{n} t_{i} \Phi\left(\mathbf{d}_{i}\right)=\Phi\left(\sum_{i=1}^{n} t_{i} \mathbf{d}_{i}\right)=\Phi(\mathbf{t})$. Therefore, if $\mathbf{t}, \mathbf{s} \in T$, we have $\Phi(\mathbf{t}-\mathbf{s})=\phi\left(u^{*}\right)-\phi\left(u^{*}\right)=0$, so $\operatorname{ker}\left(\xi^{*}\right)=\mathbb{D} \subseteq \operatorname{ker}(\Phi)$, and it follows that there exists a group homomorphism $\phi^{*}: G^{*} \rightarrow K$ such that $\phi^{*} \circ \xi^{*}=\Phi$. Consequently, $\phi^{*}\left(a_{i}^{*}\right)=\phi^{*}\left(\xi^{*}\left(\mathbf{d}_{i}\right)\right)=\Phi\left(\mathbf{d}_{i}\right)=\phi\left(a_{i}^{*}\right)$ for $i=1,2, \ldots, n$. Since every element in $E^{*}$ is a linear combination of the atoms $a_{i}^{*}$ with nonnegative integer coefficients and $\phi$ is a $K$-valued measure, it follows that the group homomorphism $\phi^{*}$ agrees with $\phi$ on $E^{*}$.

COROLLARY 2.8. The unital group $G$ is a unigroup if and only if $\operatorname{ker}(\xi) \subseteq \mathbb{D}$.

The unigroup $G^{*}$ in Theorem 2.7 is uniquely determined (up to an isomorphism of unital groups) by the structure of the effect algebra $E$, and it is called the unigroup for the effect algebra $E$ [1]. As can be seen from the proof of Theorem 2.7, the structure of $G^{*}$ is encoded in the set $T$ of multiplicity vectors, hence it is implicit in the canonical surjection $\xi$.

3. $\mathbb{Q}$-valued states. We maintain the assumptions and notation of Section 1.1 and Definition 2.3. In this section, we establish a bijective correspondence $\omega \leftrightarrow \bar{\omega}$ between $\mathbb{Q}$-valued states $\omega \in \Omega(G)$ and surjective order-preserving 
group homomorphisms $\bar{\omega}: G \rightarrow \mathbb{Z}$, and we use the mapping $\omega \mapsto \bar{\omega}$ to define an order-preserving group homomorphism $\rho$ from $G$ into a simplicial group $\mathbb{Z}^{\kappa}$.

By [3, Theorem 5.3], the state space $\Omega(G)$ is a polytope and its set of extreme points $\partial_{e}(\Omega(G))$ is a finite set of $\mathbb{Q}$-valued states. A state $\omega \in \Omega(G)$ is said to be dispersion free if and only if it takes on only the values 0 and 1 on the unit interval $E=G^{+}[0, u]$. Every dispersion-free state $\omega \in \Omega(G)$ belongs to $\partial_{e}(\Omega(G))$.

NotATion 3.1. Let $\left\{\omega_{1}, \omega_{2}, \ldots, \omega_{\kappa}\right\}:=\partial_{e}(\Omega(G))$ be the set of extreme points of the polytope $\Omega(G)$.

LEMMA 3.2. Let $\phi: G \rightarrow \mathbb{Q}$ be a group homomorphism and assume that $\phi\left(G^{+}\right) \subseteq \mathbb{Q}^{+}$(i.e., $\phi$ is order preserving). Then

(i) $0<\phi(u)$ if and only if there is at least one $i \in\{1,2, \ldots, n\}$ with $0<$ $\phi\left(a_{i}\right)$;

(ii) $\phi(u)=0$ if and only if $\phi$ is the zero homomorphism;

(iii) if $\phi(u) \neq 0$, then $\omega: G \rightarrow \mathbb{Q}$ defined by $\omega:=(1 / \phi(u)) \phi$ is a $\mathbb{Q}$-valued state.

Proof. (i) If $0<\phi\left(a_{i}\right)$, then the fact that $a_{i} \leq u$ implies $0<\phi\left(a_{i}\right) \leq$ $\phi(u)$. Conversely, suppose $0<\phi(u)$ and let $\mathbf{t}=\left(t_{1}, t_{2}, \ldots, t_{n}\right) \in T$. Then $u=$ $\sum_{i=1}^{n} t_{i} a_{i}$, whence $0<\phi(u)=\sum_{i=1}^{n} t_{i} \phi\left(a_{i}\right)$, and it follows that at least one $\phi\left(a_{i}\right)$ must be strictly positive.

(ii) Suppose $\phi(u)=0$. Then $\phi\left(a_{i}\right)=0$ for $i=1,2, \ldots, n$ by (i) and, since $\left\{a_{1}, a_{2}, \ldots, a_{n}\right\}$ is a set of generators for $G$, it follows that $\phi$ is the zero homomorphism. The converse is obvious.

(iii) Suppose $\phi(u) \neq 0$. Then $\omega=(1 / \phi(u)) \phi$ is a group homomorphism from $G$ to $\mathbb{Q}, \omega\left(G^{+}\right) \subseteq \mathbb{Q}^{+}$, and $\omega(u)=1$.

LEMMA 3.3. Let $\zeta: G \rightarrow \mathbb{Z}$ be a group homomorphism. Then the following conditions are mutually equivalent:

(i) $\zeta(G)=\mathbb{Z}$;

(ii) $\zeta^{-1}(1) \neq \varnothing$;

(iii) the nonzero integers in the list $\zeta\left(a_{1}\right), \zeta\left(a_{2}\right), \ldots, \zeta\left(a_{n}\right)$ are relatively prime.

Proof. (i) $\Leftrightarrow$ (ii). Obviously (i) $\Rightarrow$ (ii). Conversely, if (ii) holds, there exists $g_{1} \in$ $G$ with $\zeta\left(g_{1}\right)=1$, whence $\zeta\left(k g_{1}\right)=k \zeta\left(g_{1}\right)=k$ and $\zeta(G)=\mathbb{Z}$.

(ii) $\Leftrightarrow$ (iii). Suppose (ii) holds, so there exists $g_{1} \in G$ with $\zeta\left(g_{1}\right)=1$. Because $\left\{a_{1}, a_{2}, \ldots, a_{n}\right\}$ is a set of generators for $G$, there are integers $x_{1}, x_{2}, \ldots, x_{n}$ such that $g_{1}=\sum_{i=1}^{n} x_{i} a_{i}$, whence $1=\sum_{i=1}^{n} x_{i} \zeta\left(a_{i}\right)$, and (iii) follows. Conversely, if (iii) holds, there are integers $x_{1}, x_{2}, \ldots, x_{n}$ such that $1=\sum_{i=1}^{n} x_{i} \zeta\left(a_{i}\right)$ and $g_{1}:=\sum_{i=1}^{n} x_{i} a_{i} \in \zeta^{-1}(1)$.

DEFINITION 3.4. Suppose that $\phi: G \rightarrow \mathbb{Q}$ is a nonzero group homomorphism such that $\phi\left(G^{+}\right) \subseteq \mathbb{Q}^{+}$. We define the group homomorphism $\bar{\phi}: G \rightarrow \mathbb{Q}$ 
as follows: $0 \leq \phi\left(a_{i}\right)$ for $i=1,2, \ldots, n$ and $0<\phi\left(a_{i}\right)$ for at least one $i$ by Lemma 3.2. Write the strictly positive rational numbers in the list $\phi\left(a_{1}\right), \phi\left(a_{2}\right)$, $\ldots, \phi\left(a_{n}\right)$ as reduced fractions, let $q>0$ be the least common multiple of the denominators of the positive fractions in the list, and let $p>0$ be the greatest common divisor of the positive integers in the list $q \phi\left(a_{1}\right), q \phi\left(a_{2}\right), \ldots, q \phi\left(a_{n}\right)$. Define $\bar{\phi}: G \rightarrow \mathbb{Q}$ by $\bar{\phi}(g):=(q / p) \phi(g)$ for all $g \in G$.

LEMMA 3.5. Let $\phi: G \rightarrow \mathbb{Q}$ be a nonzero group homomorphism such that $\phi\left(G^{+}\right) \subseteq \mathbb{Q}^{+}$. Then

(i) $\bar{\phi}: G \rightarrow \mathbb{Z}$ is a surjective group homomorphism, $\bar{\phi}\left(G^{+}\right) \subseteq \mathbb{Z}^{+}$, and $0<$ $\bar{\phi}(u)$;

(ii) $\omega:=(1 / \bar{\phi}(u)) \bar{\phi}$ is a $\mathbb{Q}$-valued state for $G$;

(iii) if $\omega=(1 / \bar{\phi}(u)) \bar{\phi}$, then $\bar{\omega}=\bar{\phi}$;

(iv) $\bar{\phi}=\phi$ if and only if $\phi(G)=\mathbb{Z}$.

Proof. (i) By our choices of $q$ and $p$ in Definition 3.4, $\bar{\phi}\left(a_{i}\right) \in \mathbb{Z}^{+}$for $i=$ $1,2, \ldots, m$, at least one of these integers is positive, and the positive integers are relatively prime. Because $\left\{a_{1}, a_{2}, \ldots, a_{n}\right\}$ is a cone-generating set in $G$ and $\bar{\phi}\left(a_{i}\right) \in \mathbb{Z}^{+}$for $i=1,2, \ldots, n$, we have $\bar{\phi}\left(G^{+}\right) \subseteq \mathbb{Z}^{+}$, whence, since $G$ is directed, $\bar{\phi}(G) \subseteq \mathbb{Z}$. By Lemma 3.3, $\bar{\phi}(G)=\mathbb{Z}$, and by Lemma 3.2, $0<\bar{\phi}(u)$.

(ii) That $\omega=(1 / \bar{\phi}(u)) \bar{\phi}$ is a $\mathbb{Q}$-valued state follows from Lemma 3.2(iii).

(iii) We have $\omega\left(a_{i}\right)=\bar{\phi}\left(a_{i}\right) / \bar{\phi}(u)$ for $i=1,2, \ldots, n$, and the nonzero numerators of these fractions are relatively prime, whence $\bar{q}:=\bar{\phi}(u)$ is the least common multiple of denominators of the positive fractions in the list $\omega\left(a_{1}\right), \omega\left(a_{2}\right), \ldots, \omega\left(a_{n}\right)$, and $\bar{p}:=1$ is the greatest common divisor of the positive integers in the list $\bar{q} \omega\left(a_{1}\right), \bar{q} \omega\left(a_{2}\right), \ldots, \bar{q} \omega\left(a_{n}\right)$. Therefore, $\bar{\omega}\left(a_{i}\right)=$ $(\bar{q} / \bar{p}) \omega\left(a_{i}\right)=\bar{\phi}\left(a_{i}\right)$ for $i=1,2, \ldots, n$ and, since $\left\{a_{1}, a_{2}, \ldots, a_{n}\right\}$ is a set of generators for the group $G$, it follows that $\bar{\omega}=\bar{\phi}$.

(iv) If $\bar{\phi}=\phi$, then $\phi(G)=\bar{\phi}(G)=\mathbb{Z}$ by (i). Conversely, suppose $\phi(G)=\mathbb{Z}$. Then $\phi\left(a_{i}\right) \in \mathbb{Z}^{+}$for $i=1,2, \ldots, n$ and by Lemma 3.3 the positive integers in the list $\phi\left(a_{1}\right), \phi\left(a_{2}\right), \ldots, \phi\left(a_{n}\right)$ are relatively prime. Hence, $p=q=1$ in Definition 3.4, and we have $\bar{\phi}=\phi$.

THEOREM 3.6. The mapping $\omega \mapsto \bar{\omega}$ is a bijection from the set of all $\mathbb{Q}$-valued states on $G$ onto the set of all surjective order-preserving group homomorphisms $\zeta: G \rightarrow \mathbb{Z}$.

Proof. If $\omega \in \Omega(G)$ and $\omega(G) \subseteq \mathbb{Q}$, then $\bar{\omega}: G \rightarrow \mathbb{Z}$ is a surjective orderpreserving group homomorphism by Lemma 3.5(i). Let $\zeta: G \rightarrow \mathbb{Z}$ be a surjective order-preserving group homomorphism. By Lemma 3.2, $0<\zeta(u)$ and $\omega:=(1 / \zeta(u)) \zeta$ is a $\mathbb{Q}$-valued state on $G$. By Lemma $3.5(i v), \bar{\zeta}=\zeta$, so by Lemma 3.5(iii), $\bar{\omega}=\zeta$. We have only to prove that the mapping $\omega \mapsto \bar{\omega}$ is injective on the set of $\mathbb{Q}$-valued states. Thus, suppose $\omega$ is a $\mathbb{Q}$-valued state on $G$ and $\zeta=\bar{\omega}$. Then, by Definition 3.4, there is a positive rational number $\lambda$ such that $\zeta=\lambda \omega$, and it follows that $\zeta(u)=\lambda \omega(u)=\lambda \cdot 1=\lambda$, whence $\omega=(1 / \zeta(u)) \zeta$ is uniquely determined by $\zeta$. 
Evidently, a state $\omega \in \Omega(G)$ is dispersion free if and only if $\omega(G) \subseteq \mathbb{Z}$. Thus, in Theorem 3.6, the dispersion-free states (if any) on $G$ are exactly the $\mathbb{Q}$-valued states $\omega \in \Omega(G)$ such that $\bar{\omega}=\omega$.

DEFINITION 3.7. Since the extreme points $\omega_{1}, \omega_{2}, \ldots, \omega_{\kappa}$ of $\Omega(G)$ are $\mathbb{Q}$ valued states, we can define $\bar{\omega}_{i}:=\overline{\omega_{i}}$ for $i=1,2, \ldots, \kappa$. The mapping $\rho: G \rightarrow \mathbb{Z}^{\kappa}$ is defined by $\rho(g):=\left(\bar{\omega}_{1}(g), \bar{\omega}_{2}(g), \ldots, \bar{\omega}_{\kappa}(g)\right)$ for all $g \in G$. We also define $\mathbf{v}:=\rho(u) \in \mathbb{Z}^{\kappa}$.

THEOREM 3.8. (i) The mapping $\rho: G \rightarrow \mathbb{Z}^{\kappa}$ is a group homomorphism and $\rho\left(G^{+}\right) \subseteq\left(\mathbb{Z}^{+}\right)^{\kappa}$.

(ii) The element $\mathbf{v}=\left(\bar{\omega}_{1}(u), \bar{\omega}_{2}(u), \ldots, \bar{\omega}_{\kappa}(u)\right) \in\left(\mathbb{Z}^{+}\right)^{\kappa}$ is an order unit in the simplicial group $\mathbb{Z}^{\kappa}$.

(iii) With the standard positive cone $\left(\mathbb{Z}^{+}\right)^{\kappa}$ and with $\mathbf{v}$ as unit, the simplicial group $\mathbb{Z}^{\kappa}$ is a unigroup with unit interval $\left(\mathbb{Z}^{+}\right)^{\kappa}[\mathbf{0 , v}]$.

(iv) $\operatorname{ker}(\rho)=\{g \in G \mid \omega(g)=0$ for all $\omega \in \Omega(G)\}$.

(v) As an abelian group, $G$ is torsion-free if and only if $\rho: G \rightarrow \mathbb{Z}^{\kappa}$ is an injection.

Proof. (i) Clearly $\rho$ is a group homomorphism and, since each $\bar{\omega}_{i}$ maps $G^{+}$into $\mathbb{Z}^{+}$, it follows that $\rho\left(G^{+}\right) \subseteq\left(\mathbb{Z}^{+}\right)^{\kappa}$.

(ii) We have $0<\bar{\omega}_{1}(u), \bar{\omega}_{2}(u), \ldots, \bar{\omega}_{\kappa}(u)$, so all coordinates of the vector $\mathbf{v}$ are strictly positive, and it follows that $\mathbf{v}$ is an order unit in $\left(\mathbb{Z}^{+}\right)^{\kappa}$.

(iii) The standard free basis vectors

$$
(1,0,0, \ldots, 0),(0,1,0, \ldots, 0),(0,0,1, \ldots, 0), \ldots,(0,0,0, \ldots, 1)
$$

belong to $\left(\mathbb{Z}^{+}\right)^{\kappa}[\mathbf{0 , v}]$ and they form a set of generators for the positive cone $\left(\mathbb{Z}^{+}\right)^{\kappa}$. Therefore, $\mathbb{Z}^{\kappa}$ is a unital group with unit $\mathbf{v}$. Since the simplicial group $\mathbb{Z}^{\kappa}$ is lattice ordered and $\mathbf{v}$ is an order unit, $\mathbb{Z}^{\kappa}$ is a unigroup with unit $\mathbf{v}$.

(iv) Let $g \in G$. Then $\rho(g)=\mathbf{0}$ if and only if $\bar{\omega}_{i}(g)=0$ for $i=1,2, \ldots, \kappa$ if and only if $\omega_{i}(g)=0$ for $i=1,2, \ldots, \kappa$. But, since every $\omega \in \Omega(G)$ is a convex combination of $\omega_{1}, \omega_{2}, \ldots, \omega_{\kappa}$, it follows that $\omega_{i}(g)=0$ for $i=1,2, \ldots, \kappa$ if and only if $\omega(g)=0$ for all $\omega \in \Omega$.

(v) Suppose $G$ is torsion-free and let $0 \neq g \in G$. By [3, Lemma 3.2], there is a group isomorphism $\phi: G \rightarrow \mathbb{Z}^{r}$ such that $\phi\left(G^{+}\right) \subseteq\left(\mathbb{Z}^{+}\right)^{r}$, hence by [2, Lemma 3.5] there is a $\mathbb{Q}$-valued state $\omega \in \Omega(G)$ with $\omega(g) \neq 0$, so $g \notin \operatorname{ker} \rho$ by (iv). Therefore, if $G$ is torsion-free, then $\rho$ is injective. Conversely, suppose $\rho$ is injective, $k$ is a positive integer, $g \in G$, and $k g=0$. Then $k \rho(g)=\mathbf{0} \in \mathbb{Z}^{\kappa}$, so $\rho(g)=\mathbf{0}$, and therefore $g=0$. Consequently, $G$ is torsion-free.

In Theorem 3.8, the unit interval $\left(\mathbb{Z}^{+}\right)^{\kappa}[\mathbf{0}, \mathbf{v}]$ is an MV-algebra and the restriction $\left.\rho\right|_{E}$ of $\rho$ to $E$ is an effect-algebra morphism of $E$ into $\left(\mathbb{Z}^{+}\right)^{\kappa}[\mathbf{0 ,} \mathbf{v}]$.

4. The canonical injection $\rho$. We now begin to focus on the question of just when $G$ is Archimedean. If $G$ is Archimedean, then $G$ is torsion-free, so in this section and the next one we will adopt as a standing hypothesis the 
assumption that $G$ is torsion-free. Thus, by [3, Lemma 3.3], we can cast our standing hypothesis as follows.

STANDING ASSUMPTIONS AND NOTATION. In this section and the next one, we assume that $r$ is a positive integer, $G=\mathbb{Z}^{r}$ as an additive abelian group, $G$ is a unital group with unit $\mathbf{u}=\left(u_{1}, u_{2}, \ldots, u_{r}\right), G^{+} \subseteq\left(\mathbb{Z}^{+}\right)^{r}$, and the unit interval $E=G^{+}[\mathbf{0}, \mathbf{u}]$ is finite. The atoms in $E$ are denoted by $\mathbf{a}_{1}, \mathbf{a}_{2}, \ldots, \mathbf{a}_{n}$.

All of the previous results are applicable to the unital group $G$, and now we have the advantage of a representation of the elements of $G$ as vectors in $\mathbb{Z}^{r}$ in such a way that all vectors in $G^{+}$have nonnegative coordinates. By Theorem 3.8(v), $\rho: G \rightarrow \mathbb{Z}^{\kappa}$ is an order-preserving injective group homomorphism from $G$ into the simplicial group $\mathbb{Z}^{\kappa}$, whence $\rho(G)$ is a subgroup of $\mathbb{Z}^{\kappa}$ that is isomorphic (as a group) to $G$. Also, $\mathbb{Z}^{\kappa}$ is a lattice-ordered unigroup with unit $\mathbf{v}=\rho(\mathbf{u})$ and the restriction $\left.\rho\right|_{E}$ of $\rho$ to $E=G^{+}[\mathbf{0}, \mathbf{u}]$ is an injective effect-algebra morphism of $E$ into the MV-algebra $\left(\mathbb{Z}^{+}\right)^{\kappa}[\mathbf{0 , v}]$.

DEFINITION 4.1. (i) The mapping $\rho: G \rightarrow \mathbb{Z}^{\kappa}$ is called the canonical injection.

(ii) The $n \times r$ matrix over $\mathbb{Z}^{+}$with $\mathbf{a}_{1}, \mathbf{a}_{2}, \ldots, \mathbf{a}_{n}$ as its successive row vectors is denoted by $A_{0}=\left[a_{i j}\right]$.

(iii) Let $\mathbf{e}_{1}=(1,0, \ldots, 0), \mathbf{e}_{2}=(0,1, \ldots, 0), \ldots, \mathbf{e}_{r}=(0,0, \ldots, 1)$ be the standard free basis vectors for the abelian group $\mathbb{Z}^{r}$.

(iv) Because $\mathbf{a}_{1}, \mathbf{a}_{2}, \ldots, \mathbf{a}_{n}$ generate the group $G$, there are (not necessarily uniquely determined) integers $c_{i j}$ for $i=1,2, \ldots, r$ and $j=1,2, \ldots, n$ such that $\mathbf{e}_{i}=\sum_{j=1}^{n} c_{i j} \mathbf{a}_{j}$. Let $C$ be the $r \times n$ matrix $C:=\left[c_{i j}\right]$.

(v) For $j=1,2, \ldots, r$, let $\pi_{j}: \mathbb{Z}^{r} \rightarrow \mathbb{Z}$ be the projection homomorphism onto the $j$ th coordinate.

The rows of the matrix $A_{0}$ are the $n$ atoms in the unit interval $E=G^{+}[\mathbf{0}, \mathbf{u}]$. The canonical surjection $\xi: \mathbb{Z}^{n} \rightarrow G=\mathbb{Z}^{r}$ is given by the formula $\xi\left(x_{1}, x_{2}, \ldots\right.$, $\left.x_{n}\right)=\left(x_{1}, x_{2}, \ldots, x_{n}\right) A_{0}$, and $\left(t_{1}, t_{2}, \ldots, t_{n}\right) \in\left(\mathbb{Z}^{+}\right)^{n}$ is a multiplicity vector if and only if $\left(t_{1}, t_{2}, \ldots, t_{n}\right) A_{0}=\left(u_{1}, u_{2}, \ldots, u_{r}\right)$. The $r \times n$ matrix $C$ over $\mathbb{Z}$ is a left inverse for $A_{0}$, that is, $C A_{0}=\mathbf{1}_{r}=$ the $r \times r$ identity matrix. Therefore, for each $j=1,2, \ldots, r$, we have $\sum_{i=1}^{n} c_{j i} a_{i j}=1$, whence the nonzero integers in the $j$ th row of the matrix $C$ are relatively prime, as are the positive integers in the $j$ th column of $A_{0}$.

If $1 \leq j \leq r$, the projection homomorphism $\pi_{j}: G \rightarrow \mathbb{Z}$ is surjective and, owing to the fact that $G^{+} \subseteq\left(\mathbb{Z}^{+}\right)^{r}$, we have $\pi_{j}\left(G^{+}\right) \subseteq \mathbb{Z}^{+}$. Therefore, by Lemma 3.2, there exists $i$ with $1 \leq i \leq n$ such that $\pi_{j}\left(\mathbf{a}_{i}\right)=a_{i j}>0$, that is, there is at least one strictly positive integer in the $j$ th column of $A_{0}$. Also, by Lemma 3.2, $\pi_{j}(\mathbf{u})=u_{j}>0$ and, by Theorem 3.6, there is a uniquely determined $\mathbb{Q}$-valued state $\gamma_{j} \in \Omega(G)$ such that $\overline{\gamma_{j}}=\pi_{j}$. Evidently, $\gamma_{j}=\left(1 / u_{j}\right) \pi_{j}$. The $\mathbb{Q}$-valued states $\gamma_{1}, \gamma_{2}, \ldots, \gamma_{r}$, which correspond to the columns of the matrix $A_{0}$, form a separating set of states for $G$.

LEMMA 4.2. Every state $\omega \in \Omega(G)$ is a unique affine linear combination of the states $\gamma_{j}:=\left(1 / u_{j}\right) \pi_{j}$ for $j=1,2, \ldots, r$. 
Proof. For $i, j=1,2, \ldots, r$, we have $\gamma_{j}\left(\mathbf{e}_{i}\right)=\left(1 / u_{j}\right) \pi_{j}\left(\mathbf{e}_{i}\right)=\left(1 / u_{j}\right) \delta_{i j}$, where $\delta_{i j}$ is the Kronecker delta. Let $\omega \in \Omega(G)$ and let $s_{i}:=u_{i} \omega\left(\mathbf{e}_{i}\right)=\omega\left(u_{i} \mathbf{e}_{i}\right)$ for $i=1,2, \ldots, r$. Then $\sum_{i=1}^{r} s_{i}=\omega(\mathbf{u})=1$. Also $\phi:=\sum_{j=1}^{r} s_{j} \gamma_{j}$ is a group homomorphism $\phi: G \rightarrow \mathbb{R}$, and $\phi\left(\mathbf{e}_{i}\right)=\sum_{i=1}^{r}\left(s_{j} / u_{j}\right) \delta_{i j}=s_{i} / u_{i}=\omega\left(\mathbf{e}_{i}\right)$ for $i=1,2, \ldots, r$. Since the group homomorphisms $\phi$ and $\omega$ agree on the free basis $\mathbf{e}_{1}, \mathbf{e}_{2}, \ldots, \mathbf{e}_{r}$ for $G$, it follows that $\omega=\phi$, and $\omega$ is an affine linear combination of the states $\gamma_{j}$ for $j=1,2, \ldots, r$. To prove that the coefficients are uniquely determined by $\omega$, suppose $\omega=\sum_{j=1}^{r} h_{j} \gamma_{j}$ with $h_{j} \in \mathbb{R}$ for $j=1,2, \ldots, r$. Then $\omega\left(\mathbf{e}_{i}\right)=\sum_{j=1}^{r} h_{j}\left(1 / u_{j}\right) \delta_{i j}=h_{i} / u_{i}$, whence $h_{i}=s_{i}$ for $i=1,2, \ldots, r$.

Since $\left\{\mathbf{a}_{i} \mid i=1,2, \ldots, n\right\}$ is a finite set of generators for $G$, it follows that $\left\{\rho\left(\mathbf{a}_{i}\right) \mid i=1,2, \ldots, n\right\}$ is a finite set of generators for $\rho(G) \subseteq \mathbb{Z}^{\kappa}$. This observation brings us to our next definition.

DEFINITION 4.3. (i) Let $\mathbf{w}_{i}:=\rho\left(\mathbf{a}_{i}\right)=\left(\bar{\omega}_{1}\left(\mathbf{a}_{i}\right), \bar{\omega}_{2}\left(\mathbf{a}_{i}\right), \ldots, \bar{\omega}_{\kappa}\left(\mathbf{a}_{i}\right)\right) \in \mathbb{Z}^{\kappa}$ for $i=1,2, \ldots, n$.

(ii) Let $W:=\left[w_{i j}\right]$ be the $n \times \kappa$ matrix with the vectors $\mathbf{w}_{1}, \mathbf{w}_{2}, \ldots, \mathbf{w}_{n}$ as its successive rows.

(iii) Let $P=\left[p_{i j}\right]$ be the $r \times \kappa$ matrix over $\mathbb{Z}$ given by the product $P:=C W$.

Since $\mathbf{0} \leq \mathbf{a}_{i} \leq \mathbf{u}$ for $i=1,2, \ldots, n$, it follows that $\mathbf{0} \leq \rho\left(\mathbf{a}_{i}\right) \leq \rho(\mathbf{u})$, whence $\mathbf{w}_{i} \in\left(\mathbb{Z}^{+}\right)^{\kappa}[\mathbf{0}, \mathbf{v}]$ for $i=1,2, \ldots, n$. By Definition 4.3(ii),

$$
w_{i j}=\bar{\omega}_{j}\left(\mathbf{a}_{i}\right) \text { for } i=1,2, \ldots, n, j=1,2, \ldots, \kappa
$$

The columns of the matrix $W$ correspond to the extreme points $\omega_{1}, \omega_{2}, \ldots, \omega_{\kappa}$ of $\Omega(G)$ and its row vectors $\mathbf{w}_{i}, i=1,2, \ldots, n$, generate the subgroup $\rho(G)$ of $\mathbb{Z}^{\kappa}$. By part (ii) of Theorem 4.4, the canonical injection $\rho$ corresponds to right multiplication of vectors in $G=\mathbb{Z}^{r}$ by the $r \times \kappa$ matrix $P$. By part (v) of the theorem, the columns of the matrix $P=C W$ are coefficients of homogeneous linear inequalities over $\mathbb{Z}$ that must be satisfied by vectors in the positive cone $G^{+}$.

THEOREM 4.4. Let $\mathbf{y}=\left(y_{1}, y_{2}, \ldots, y_{r}\right) \in G=\mathbb{Z}^{r}$. Then

(i) $\bar{\omega}_{j}(\mathbf{y})=\sum_{i=1}^{r} y_{i} p_{i j}$ for $j=1,2, \ldots, \kappa$;

(ii) $\rho(\mathbf{y})=\mathbf{y} P$;

(iii) $\rho(\mathbf{y}) \in\left(\mathbb{Z}^{+}\right)^{\kappa} \Leftrightarrow 0 \leq \sum_{i=1}^{r} p_{i j} y_{i}$ for $j=1,2, \ldots, \kappa$;

(iv) $\rho(\mathbf{y}) \in\left(\mathbb{Z}^{+}\right)^{\kappa} \Leftrightarrow 0 \leq \omega(\mathbf{y})$ for all $\omega \in \Omega(G)$;

(v) $\mathbf{y} \in G^{+} \Rightarrow 0 \leq \sum_{i=1}^{r} p_{i j} y_{i}$ for $j=1,2, \ldots, \kappa$.

Proof. (i) We have $\mathbf{y}=\sum_{i=1}^{r} y_{i} \mathbf{e}_{i}=\sum_{i=1}^{r} y_{i} \sum_{k=1}^{n} c_{i k} \mathbf{a}_{k}$, whence, for $j=1$, $2, \ldots, \kappa$

$$
\bar{\omega}_{j}(\mathbf{y})=\sum_{i=1}^{r} \sum_{k=1}^{n} y_{i} c_{i k} \bar{\omega}_{j}\left(\mathbf{a}_{k}\right)=\sum_{i=1}^{r} y_{i} \sum_{k=1}^{n} c_{i k} w_{k j}=\sum_{i=1}^{r} y_{i} p_{i j} .
$$

Parts (ii) and (iii) follow from (i) and the definition of $\rho$. 
(iv) Suppose $\rho(\mathbf{y}) \in\left(\mathbb{Z}^{+}\right)^{\kappa}$. Then $0 \leq \bar{\omega}_{i}(\mathbf{y})$, so $0 \leq \omega_{i}(\mathbf{y})$ for $i=1,2, \ldots, \kappa$. If $\omega \in \Omega(G)$, then $\omega$ is a convex combination of $\omega_{1}, \omega_{2}, \ldots, \omega_{\kappa}$, and it follows that $0 \leq \omega(\mathbf{y})$. Conversely, if $0 \leq \omega(\mathbf{y})$ for all $\omega \in \Omega(G)$, then $0 \leq \omega_{i}(\mathbf{y})$, so $0 \leq \bar{\omega}_{i}(\mathbf{y})$ for $i=1,2, \ldots \kappa$, whence $\rho(\mathbf{y}) \in\left(\mathbb{Z}^{+}\right)^{\kappa}$.

(v) It follows from (iii) and the fact that $\rho\left(G^{+}\right) \subseteq\left(\mathbb{Z}^{+}\right)^{\kappa}$.

5. The Archimedean property. We maintain the hypotheses and notation of Section 4. The canonical surjection $\xi$ and the canonical injection $\rho$ are orderpreserving group homomorphisms

$$
\mathbb{Z}^{n} \stackrel{\xi}{\longrightarrow} G \stackrel{\rho}{\longrightarrow} \mathbb{Z}^{\kappa}
$$

that link the torsion-free unital group $G$ with the simplicial groups $\mathbb{Z}^{n}$ and $\mathbb{Z}^{\kappa}$ and that satisfy

$$
\mathbf{d}_{i} \stackrel{\xi}{\longmapsto} \mathbf{a}_{i} \stackrel{\rho}{\longmapsto} \mathbf{w}_{i} \text { for } i=1,2, \ldots, n
$$

as well as

$$
G^{+}=\xi\left(\left(\mathbb{Z}^{+}\right)^{n}\right), \quad \rho\left(G^{+}\right) \subseteq\left(\mathbb{Z}^{+}\right)^{\kappa} \cap \rho(G) .
$$

As attractive as the setup in (5.1), (5.2), and (5.3) may be, there is an obvious asymmetry in (5.3). Indeed, although $\xi$ determines the positive cone in $G$ via $G^{+}=\xi\left(\left(\mathbb{Z}^{+}\right)^{n}\right), \rho$ does not necessarily determine $G^{+}$. For symmetry, one would like to have

$$
G^{+}=\rho^{-1}\left(\left(\mathbb{Z}^{+}\right)^{\kappa}\right) .
$$

Evidently, (5.4) holds if and only if the inclusion in (5.3) is an equality, that is, if and only if

$$
\rho\left(G^{+}\right)=\left(\mathbb{Z}^{+}\right)^{\kappa} \cap \rho(G)
$$

and condition (5.5) is equivalent to the requirement that, for $\mathbf{a}, \mathbf{b} \in G$,

$$
\mathbf{a} \leq \mathbf{b} \text { in } G \Longleftrightarrow \rho(\mathbf{a}) \leq \rho(\mathbf{b}) \text { in the simplicial group } \mathbb{Z}^{\kappa} \text {. }
$$

The image $\rho(G)$ of $G$ under $\rho$ is the subgroup of $\mathbb{Z}^{\kappa}$ generated by the vectors $\mathbf{w}_{1}, \mathbf{w}_{2}, \ldots, \mathbf{w}_{n}$, and it can be organized into a partially ordered abelian group in either of the following two natural ways.

(i) Use the restriction to $\rho(G)$ of the partial order on the simplicial group $\mathbb{Z}^{\kappa}$. In this case, the positive cone for $\rho(G)$ is the induced positive cone $(\rho(G))^{+}$ $=\rho(G) \cap\left(\mathbb{Z}^{+}\right)^{\kappa}$.

(ii) Partially order $\rho(G)$ with the subcone $(\rho(G))^{+}=\rho\left(G^{+}\right)$of the induced positive cone $\rho(G) \cap\left(\mathbb{Z}^{+}\right)^{\kappa}$ as its positive cone. In this case, $\rho(G)$ is a unital group with unit $\mathbf{v}=\rho(\mathbf{u})$ and is isomorphic as a unital group to $G$ under $\rho$ : $G \rightarrow \rho(G)$. 
The equivalent conditions (5.4), (5.5), and (5.6) are themselves equivalent to the requirement that these two partial orders are the same. By the following theorem, all of these conditions are equivalent to the condition that $G$ is Archimedean, or equivalently, that $\Omega(G)$ is cone determining.

THEOREM 5.1. The following conditions are mutually equivalent:

(i) $G^{+}=\rho^{-1}\left(\left(\mathbb{Z}^{+}\right)^{\kappa}\right)$;

(ii) $G$ is Archimedean;

(iii) $\Omega(G)$ is cone determining, that is, $G^{+}=\{\mathbf{y} \in G \mid 0 \leq \omega(\mathbf{y})$ for all $\omega \in$ $\Omega(G)\}$;

(iv) $G^{+}=\left\{\left(y_{1}, y_{2}, \ldots, y_{r}\right) \in \mathbb{Z}^{r} \mid 0 \leq \sum_{i=1}^{r} p_{i j} y_{i}\right.$ for $\left.j=1,2, \ldots, \kappa\right\}$;

(v) the positive cone $G^{+}$is determined by a finite system of homogeneous linear inequalities over $\mathbb{Z}$, that is, there exists an $r \times s$ matrix $\left[b_{i j}\right]$ over $\mathbb{Z}$ such that $G^{+}=\left\{\left(y_{1}, y_{2}, \ldots, y_{r}\right) \in \mathbb{Z}^{r} \mid 0 \leq \sum_{i=1}^{r} b_{i j} y_{i}\right.$ for $\left.j=1,2, \ldots, s\right\}$.

Proof. (i) $\Rightarrow$ (ii). Assume that (i) holds. The abelian group $G$ is isomorphic under $\rho: G \rightarrow \rho(G)$ to the subgroup $\rho(G)$ of $\mathbb{Z}^{\kappa}$. Organize $\rho(G)$ into a partially ordered abelian group under the restriction to $\rho(G)$ of the standard partial order on $\mathbb{Z}^{\kappa}$. Then $\rho(G)$ inherits the Archimedean property from the simplicial group $\mathbb{Z}^{\kappa}$. Also, condition (i) is equivalent to the requirement that the isomorphism $\rho: G \rightarrow \rho(G)$ is an isomorphism of partially ordered abelian groups, whence $G$ acquires the Archimedean property.

(ii) $\Leftrightarrow$ (iii). Follows from [7, Theorem 4.14].

(iii) $\Leftrightarrow$ (iv). Follows from parts (iii) and (iv) of Theorem 4.4.

(iv) $\Rightarrow$ (i). Follows from Theorem 4.4(iv).

(iv) $\Rightarrow(v)$. This is obvious.

(v) $\Rightarrow$ (iii). Assume (v) and for $j=1,2, \ldots, s$ define the group homomorphism $\phi_{j}: G \rightarrow \mathbb{Z}$ by $\phi_{j}(\mathbf{y}):=\sum_{i=1}^{r} b_{i j} y_{i}$ for $\mathbf{y}=\left(y_{1}, y_{2}, \ldots, y_{r}\right) \in G=\mathbb{Z}^{r}$. By $(\mathrm{v})$, we have $\phi_{j}\left(G^{+}\right) \subseteq \mathbb{Z}^{+}$for $j=1,2, \ldots, s$. By dropping all occurrences (if any) of the zero homomorphism from the list $\phi_{1}, \phi_{2}, \ldots, \phi_{s}$, we can and do assume that $\phi_{j} \neq 0$ for $j=1,2, \ldots, s$. For $j=1,2, \ldots, s$, define $\bar{\phi}_{j}:=\overline{\phi_{j}}$ as in Definition 3.4. By Lemma 3.5(i), $\bar{\phi}_{j}: G \rightarrow \mathbb{Z}$ is a surjective order-preserving group homomorphism for $j=1,2, \ldots, s$. Furthermore, by (i), $G^{+}=\left\{\mathbf{y} \in G \mid 0 \leq \bar{\phi}_{j}(\mathbf{y})\right.$ for $j=$ $1,2, \ldots, s\}$. By Lemma 3.5(ii), $v_{j}:=\left(1 / \bar{\phi}_{j}(\mathbf{u})\right) \bar{\phi}_{j}$ is a $\mathbb{Q}$-valued state in $\Omega(G)$ for $j=1,2, \ldots, s$. Therefore, by (i), $G^{+}=\left\{\mathbf{y} \in G \mid 0 \leq v_{j}(\mathbf{y})\right.$ for $\left.j=1,2, \ldots, s\right\}$, from which (iii) follows.

COROLlaRY 5.2. If $G$ is Archimedean, then the restriction $\left.\rho\right|_{E}$ of $\rho$ to $E$ embeds the effect algebra $E$ into the $M V$-algebra $\left(\mathbb{Z}^{+}\right)^{\kappa}[\mathbf{0 , v}]$ as a subeffect algebra $\rho(E)$.

To help fix ideas, we present a very simple example with $n=3, r=2$, and $\kappa=2$ to illustrate some of the ideas developed in this paper.

EXAMPLE 5.3. Let $G=\mathbb{Z}^{2}$ as an additive abelian group and define $\mathbf{a}_{i} \in G$ for $i=1,2,3$ by $\mathbf{a}_{1}:=(1,0), \mathbf{a}_{2}:=(1,1)$, and $\mathbf{a}_{3}:=(1,2)$. Let $\xi: \mathbb{Z}^{3} \rightarrow G$ be defined by 
$\xi\left(x_{1}, x_{2}, x_{3}\right):=\sum_{i=1}^{3} x_{i} \mathbf{a}_{i}$, and let $G^{+}:=\xi\left(\left(\mathbb{Z}^{+}\right)^{3}\right)=\left\{\left(x_{1}+x_{2}+x_{3}, x_{2}+2 x_{3}\right) \mid\right.$ $\left.x_{1}, x_{2}, x_{3} \in \mathbb{Z}^{+}\right\}$. Then $G^{+}+G^{+} \subseteq G^{+}$and $-G^{+} \cap G^{+}=\{\mathbf{0}\}$, so $G$ can be organized into a partially ordered abelian group with positive cone $G^{+}$. Define $\mathbf{u}:=(3,2) \in G$. Suppose $\left(y_{1}, y_{2}\right) \in G$, choose a positive integer $k$ larger than both $y_{1}-y_{2}$ and $y_{2} / 2$, and let $x_{1}:=k+y_{2}-y_{1}, x_{2}:=2 k-y_{2}$, and $x_{3}=0$. Then $\left(x_{1}, x_{2}, x_{3}\right) \in\left(\mathbb{Z}^{+}\right)^{3}$ and $\xi\left(x_{1}, x_{2}, x_{3}\right)=\left(3 k-y_{1}, 2 k-y_{2}\right)=k(3,2)-\left(y_{1}, y_{2}\right)$, whence $\left(y_{1}, y_{2}\right) \leq k \mathbf{u}$. Therefore, $\mathbf{u}$ is an order unit in $G$. By direct computation, $E:=G^{+}[\mathbf{0}, \mathbf{u}]$ consists of the eight elements $\mathbf{0}, \mathbf{u}, \mathbf{a}_{1}, \mathbf{a}_{2}, \mathbf{a}_{3}, \mathbf{u}-\mathbf{a}_{1}, \mathbf{u}-\mathbf{a}_{2}$, and $\mathbf{u}-\mathbf{a}_{3}$. Also, $\mathbf{a}_{1}, \mathbf{a}_{2}, \mathbf{a}_{3}$ are the atoms in $E, G$ is a unital group with unit $\mathbf{u}$, $E$ is the unit interval in $G$, and $\xi: \mathbb{Z}^{3} \rightarrow G$ is the canonical surjection.

There are exactly two multiplicity vectors in $T=\xi^{-1}(\mathbf{u}) \cap\left(\mathbb{Z}^{+}\right)^{3}$, namely, $\mathbf{t}_{1}:=$ $(2,0,1)$ and $\mathbf{t}_{2}:=(1,2,0)$. Also $\operatorname{ker}(\xi)=\{x(1,-2,1) \mid x \in \mathbb{Z}\}$, so $\mathbb{D}=\operatorname{ker}(\xi)$, and it follows from Corollary 2.8 that $G$ is a unigroup.

We have

$$
A_{0}=\left[\begin{array}{ll}
1 & 0 \\
1 & 1 \\
1 & 2
\end{array}\right]
$$

and $C=\left[\begin{array}{ccc}1 & 0 & 0 \\ -1 & 1 & 0\end{array}\right]$ is a left inverse over $\mathbb{Z}$ for $A_{0}$. The state space $\Omega(G)$ is onedimensional and has two extreme points $\omega_{1}$ and $\omega_{2}$, where $\omega_{1}\left(y_{1}, y_{2}\right)=$ $(1 / 4)\left(2 y_{1}-y_{2}\right)$ and $\omega_{2}\left(y_{1}, y_{2}\right)=y_{2} / 2$ for $\left(y_{1}, y_{2}\right) \in G$. Hence,

$$
W=\left[\begin{array}{ll}
2 & 0 \\
1 & 1 \\
0 & 2
\end{array}\right], \quad P=C W=\left[\begin{array}{cc}
2 & 0 \\
-1 & 1
\end{array}\right]
$$

Thus, the canonical injection $\rho: G \rightarrow \mathbb{Z}^{2}$ is given by $\rho\left(y_{1}, y_{2}\right)=\left(y_{1}, y_{2}\right) P=$ $\left(2 y_{1}-y_{2}, y_{2}\right)$ for $\left(y_{1}, y_{2}\right) \in \mathbb{Z}^{2}=G$. The two columns of $P$ encode two homogeneous linear inequalities that must be satisfied by all $\left(y_{1}, y_{2}\right) \in G^{+}$, namely, $0 \leq 2 y_{1}-y_{2}$ and $0 \leq y_{2}$, that is, $0 \leq y_{2} \leq 2 y_{1}$. Computation reveals that, conversely, $0 \leq y_{2} \leq 2 y_{1} \Rightarrow\left(y_{1}, y_{2}\right) \in G^{+}$, whence $G$ is Archimedean by Theorem 5.1.

The order unit $\mathbf{v}=\rho(\mathbf{u})$ in the simplicial group $\mathbb{Z}^{2}$ is $\mathbf{v}=(4,2)$, and the MValgebra $\left(\mathbb{Z}^{+}\right)^{2}[(0,0),(4,2)]=\left\{\left(z_{1}, z_{2}\right) \in \mathbb{Z}^{2} \mid 0 \leq z_{1} \leq 4\right.$ and $\left.0 \leq z_{2} \leq 2\right\}$ with coordinatewise partial order. The effect-algebra morphism $\left.\rho\right|_{E}$ embeds $E$ into the MV-algebra $\left(\mathbb{Z}^{+}\right)^{2}[(0,0),(4,2)]$ in such a way that $\mathbf{a}_{1} \mapsto(2,0), \mathbf{a}_{2} \mapsto(1,1)$, and $\mathbf{a}_{3} \mapsto(0,2)$.

\section{REFERENCES}

[1] M. K. Bennett and D. J. Foulis, Interval and scale effect algebras, Adv. in Appl. Math. 19 (1997), no. 2, 200-215.

[2] D. J. Foulis, Removing the torsion from a unital group, to appear in Rep. Math. Phys. 
[3] _ _ Representation of a unital group with a finite unit interval, to appear in Demonstratio Math.

[4] _ MV and Heyting effect algebras, Found. Phys. 30 (2000), no. 10, 1687-1706.

[5] D. J. Foulis and M. K. Bennett, Effect algebras and unsharp quantum logics, Found. Phys. 24 (1994), no. 10, 1331-1352.

[6] D. J. Foulis, R. J. Greechie, and M. K. Bennett, The transition to unigroups, Internat. J. Theoret. Phys. 37 (1998), no. 1, 45-63.

[7] K. R. Goodearl, Partially Ordered Abelian Groups with Interpolation, Mathematical Surveys and Monographs, vol. 20, American Mathematical Society, Rhode Island, 1986.

[8] M. A. Perles, On Dilworth's theorem in the infinite case, Israel J. Math. 1 (1963), 108-109.

David J. Foulis: Department of Mathematics and Statistics, University of Massachusetts, Amherst, MA 01003, USA

E-mail address: foulis@math.umass.edu 


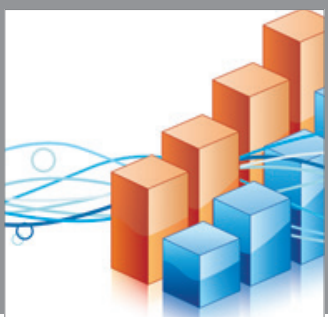

Advances in

Operations Research



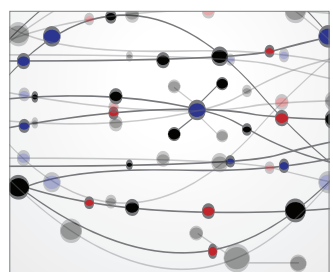

\section{The Scientific} World Journal


International Journal of

Mathematics and

Mathematical

Sciences
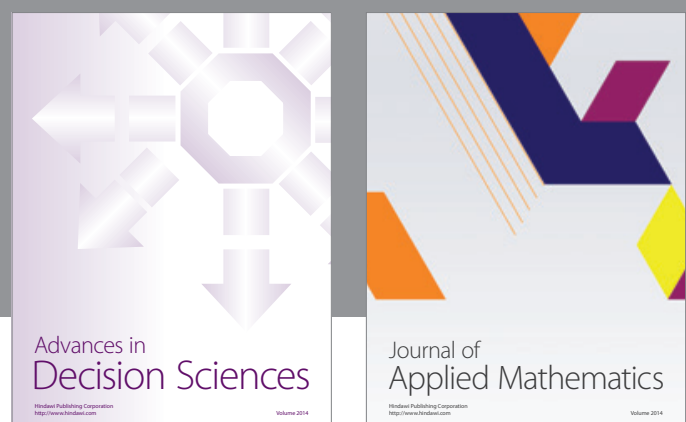

Journal of

Applied Mathematics
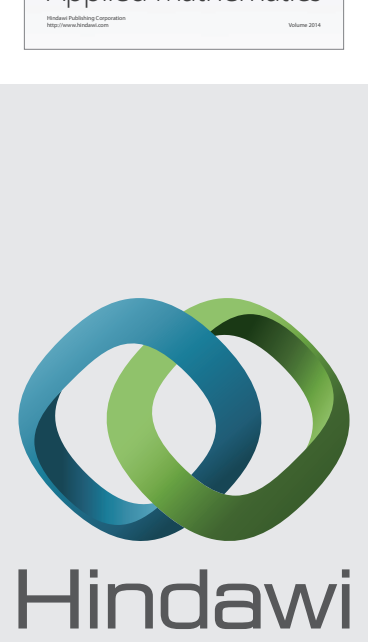

Submit your manuscripts at http://www.hindawi.com


Mathematical Problems in Engineering
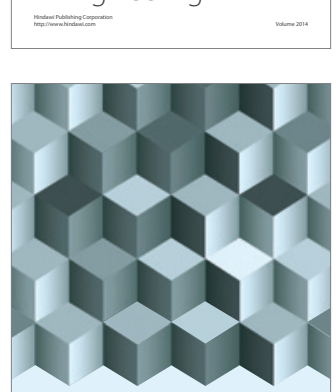

Journal of

Function Spaces
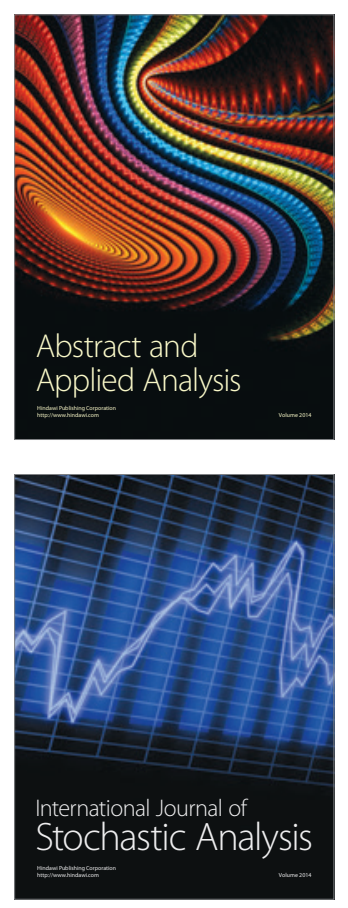

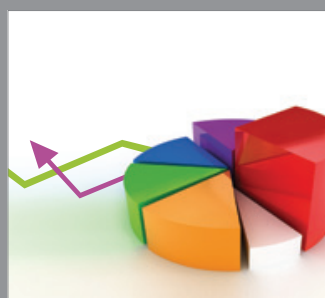

ournal of

Probability and Statistics

Promensencen
